\title{
Three-dimensional dose reconstruction-based pretreatment dosimetric verification in volumetric modulated arc therapy for prostate cancer
}

\author{
Yuri Jeong*, Jeong Geun Oh*, Jeong Ku Kang, Sun Rock Moon, Kang Kyoo Lee \\ Department of Radiation Oncology, Wonkwang University Hospital, Wonkwang University School of Medicine, Iksan, Korea
}

Received: February 3, 2020

Revised: March 9, 2020

Accepted: March 10, 2020

Correspondence:

Kang Kyoo Lee,

Department of Radiation Oncology,

Wonkwang University Hospital,

Wonkwang University School of

Medicine, 895 Muwang-ro, Iksan

54538, Korea.

Tel: +82-63-859-1882

Fax: +82-63-859-1889

E-mail:kklee@wku.ac.kr

ORCID:

https://orcid.org/0000-0003-0573-3994

"These authors contributed equally to this work.
Purpose: We performed three-dimensional (3D) dose reconstruction-based pretreatment verification to evaluate gamma analysis acceptance criteria in volumetric modulated arc therapy (VMAT) for prostate cancer.

Materials and Methods: Pretreatment verification for 28 VMAT plans for prostate cancer was performed using the COMPASS system with a dolphin detector. The 3D reconstructed dose distribution of the treatment planning system calculation (TC) was compared with that of COMPASS independent calculation (CC) and COMPASS reconstruction from the dolphin detector measurement (CR). Gamma results (gamma failure rate and average gamma value [GFR and $\gamma \mathrm{Avg}$ ]) and dose-volume histogram (DVH) deviations, 98\%, $2 \%$ and mean dose-volume difference $\left(\mathrm{DD}_{98 \%,} \mathrm{DD}_{2 \%}\right.$ and $\left.\mathrm{DD}_{\text {mean }}\right)$, were evaluated. Gamma analyses were performed with two acceptance criteria, 2\%/2 mm and 3\%/3 mm.

Results: The GFR in 2\%/2 mm criteria were less than $8 \%$, and those in $3 \% / 3 \mathrm{~mm}$ criteria were less than $1 \%$ for all structures in comparisons between TC, CC, and CR. In the comparison between TC and $\mathrm{CR}, \mathrm{GFR}$ and $\gamma \mathrm{Avg}$ in $2 \% / 2 \mathrm{~mm}$ criteria were significantly higher than those in 3\%/3 mm criteria. The DVH deviations were within $2 \%$, except for $\mathrm{DD}_{\text {mean }}(\%)$ for rectum and bladder.

Conclusions: The $3 \% / 3 \mathrm{~mm}$ criteria were not strict enough to identify any discrepancies between planned and measured doses, and DVH deviations were less than $2 \%$ in most parameters. Therefore, gamma criteria of $2 \% / 2 \mathrm{~mm}$ and DVH related parameters could be a useful tool for pretreatment verification for VMAT in prostate cancer.

Keywords: Prostatic neoplasms, Intensity-modulated radiotherapy, Pretreatment verification, Gamma analysis, Acceptance criteria, Dose-volume histogram

\section{Introduction}

External beam radiation therapy is one of the main treatment options for prostate cancer [1]. Intensity-modulated radiotherapy (IMRT), which has been widely used for prostate cancer, can provide conformal target coverage while minimizing the dose to adjacent organs [2]. Volumetric modulated arc therapy (VMAT) is an IMRT technique that delivers rotational cone beams with simultaneous modulation of multileaf collimator (MLC) leaves and dose rates [3]. Compared with IMRT which uses fixed gantry angles, VMAT provides an extra degree of freedom, and can reduce treat- ment delivery time and monitor units (MUs) while providing equivalent or improved dose distributions $[4,5]$. However, because of its complexities in treatment planning and treatment delivery systems, VMAT requires a stringent pretreatment verification to check whether the intended doses will be delivered accurately.

Gamma analysis, introduced by Low et al. [6] in 1998, has been predominantly used for pretreatment verification [7-10]. It compares reference and evaluated dose distributions based on a combination of dose difference and distance-to- agreement (DTA) criteria and calculates gamma index $(\gamma)$ and gamma passing/failure rates. Gamma failure rate (GFR), which is the percentage of points

Copyright@ 2020 The Korean Society for Radiation Oncology

This is an Open Access article distributed under the terms of the Creative Commons Attribution Non-Commercial License (http://creativecommons.org/licenses/by-nc/4.0/) which permits unrestricted non-commercial use, distribution, and reproduction in any medium, provided the original work is properly cited. 
with $\gamma>1$, has been used as a tool to decide whether or not a treatment plan is acceptable. Many studies, including AAPM TG218 , have made efforts to develop recommendations on the choice of acceptance criteria for dose difference/DTA as well as the tolerance and action limits for gamma passing/failure rates [6-10]. However, whether the GFR is sufficient to detect clinically relevant error is unknown, as this measure only focusses on the quantity of error. In addition, recommendations for gamma analysis are not cancer- or structure-specific. Although three-dimensional (3D) dose reconstruction-based pretreatment verification is available, no recommendation regarding its interpretation and incorporation into choosing acceptance criteria for gamma analysis have been made.

In this study, we performed 3D dose reconstruction-based pretreatment verification to evaluate gamma analysis acceptance criteria in VMAT for prostate cancer.

\section{Materials and Methods}

Pretreatment verifications for 28 VMAT plans for prostate cancer patients treated between May 2017 and April 2018 were performed using the COMPASS system in conjunction with a dolphin detector (IBA Dosimetry GmbH, Schwarzenbruck, Germany). This study was approved by the Institutional Review Board of Wonkwang University Hospital (WKUH 2018-12-004). The informed consent was waived.

\section{Simulation and VMAT plan}

Each patient was immobilized with a dual leg positioner in the supine position, and computed tomography (CT) images from the second lumbar vertebra to the proximal half of the femur with a 3-mm slice thickness were obtained. Patients were instructed to void, drink $500 \mathrm{~mL}$ of water, and then wait for 30 minutes before the acquisition of CT images as well as before each fraction of treatment. Clinical target volume (CTV) was defined as the surgical bed of prostate and seminal vesicles in adjuvant and salvage radiotherapy. In cases of definitive radiotherapy ( $n=5)$, CTV included prostate gland and seminal vesicles on the planning CT. Planning target volume (PTV) was defined as expansion of CTV with a margin of $5 \mathrm{~mm}$ in the other direction, except for the rectum, where a 3-mm margin was used. Organs at risk (OARs), including rectum, bladder, both femur heads, and sigmoid colon were also contoured. The PTV dose of each patient was prescribed as $66 \mathrm{~Gy}$, $70 \mathrm{~Gy}$, and $76 \mathrm{~Gy}$ as the adjuvant, salvage, and definitive aim in 2 Gy fractions, respectively.

All VMAT plans were created using two-arc with a 6-MV photon beam and a maximum dose rate of $600 \mathrm{MU} / \mathrm{min}$ using a VitalBeam linear accelerator mounted with a millennium 120 MLC sys- tem (Varian Medical System, Palo Alto, CA, USA). The first arc beam (clockwise rotation) ranged from $181^{\circ}$ to $179^{\circ}$, and the second arc beam (counterclockwise rotation) ranged from $179^{\circ}$ to $181^{\circ}$. The collimator angles were $330^{\circ}$ and $30^{\circ}$ for the first and second arc beam, respectively. The plan objectives were as followings: (1) the prescribed dose was at least 95\% of PTV and at least $99 \%$ of CTV; (2) a maximum dose $\left(D_{\max }\right)$ of $<40$ Gy to the sigmoid colon and both femur heads; ( 3 ) a mean dose $\left(D_{\text {mean }}\right)$ of $<18$ Gy to the femur; (4) the volume of the rectum irradiated by $68 \mathrm{~Gy}, 65$ $\mathrm{Gy}, 60 \mathrm{~Gy}$, and $50 \mathrm{~Gy}\left(\mathrm{~V}_{68 \mathrm{~Gy}}, \mathrm{~V}_{65 \mathrm{~Gy}}, \mathrm{~V}_{60 \mathrm{~Gy}}\right.$, and $\left.\mathrm{V}_{50 \mathrm{~Gy}}\right)$ to $<15 \%$, <25\%, $<35 \%$, and $<50 \%$, respectively; and (5) the $V_{62 G y}$ of the bladder was $<40 \%$. Optimization was performed using an Eclipse photon optimizer (version 13.7.14), and dose calculations were performed using the Anisotropic Analytic Algorithm (version 13.7.14) with a grid size of $2.5 \mathrm{~mm}$.

\section{Pretreatment verification and statistics}

The pretreatment verification was performed using a COMPASS system with a dolphin detector. First, a DICOM dataset consisting of CT data, structure, plan, and dose was exported from the treatment planning system (TPS) to the COMPASS system. Second, an independent calculation was done using a collapsed cone algorithm with a grid size of $2.5 \mathrm{~mm}$ (COMPASS dose engine, version 4.0). Third, the measurement was done using the dolphin detector, a two-dimensional array with 1,513 ionization chambers, according to the manufacturer guidelines (COMPASS 4.0 user's guide): (1) the detector was mounted on the gantry of the VitalBeam; (2) the detector setup procedure was performed, including pre-irradiation (1000 MU at field size of $40 \mathrm{~cm} \times 40 \mathrm{~cm}$ ), background measurement (100 MU at field size of $10 \mathrm{~cm} \times 10 \mathrm{~cm}$ ), and subsequent absolute calibration; (3) the detector was connected to the COMPASS system, and the gantry angle sensor was tested; (4) the measurement was performed and assigned to the plan; (5) the measurement was exported; and (6) 3D reconstructed dose distribution from the dolphin detector was calculated with the COMPASS dose engine.

Fig. 1 shows a representative case of pretreatment verification using the COMPASS system with the dolphin detector. The 3D reconstructed dose distribution of the TPS calculation (TC) was compared with that of COMPASS independent calculation (CC) and COMPASS reconstruction from the dolphin detector measurement (CR), and the gamma results and dose-volume histogram (DVH) deviations were evaluated for each structure, PTV, CTV, as well as rectum and bladder. Gamma results, including GFR and average gamma value ( $\gamma \mathrm{Avg}$ ) were automatically calculated according to the equations defined by Low et al. [6]. Global normalization was used, and gamma results with gamma criteria of $2 \% / 2 \mathrm{~mm}$ were 

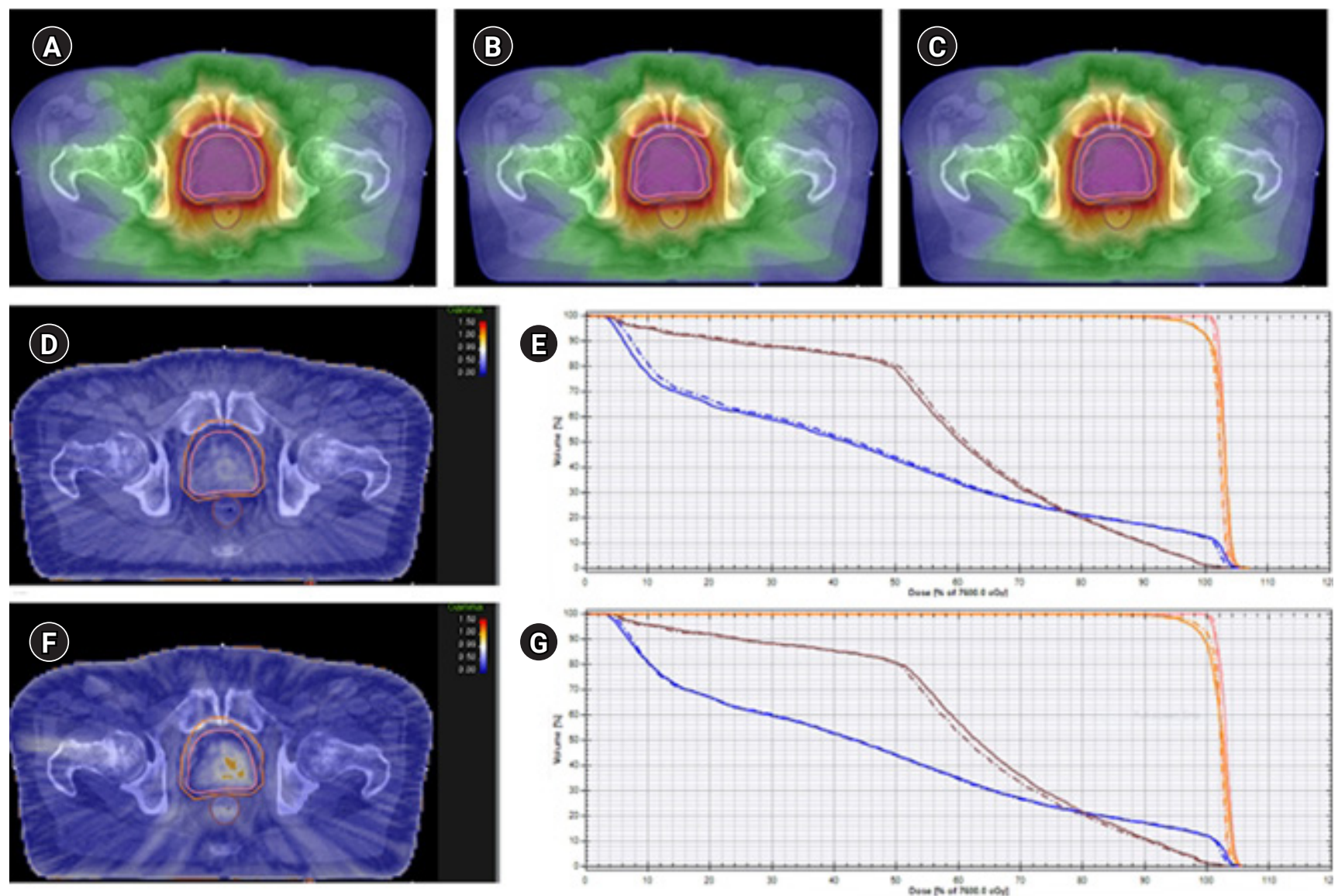

Fig. 1. A representative case of pretreatment verification by COMPASS system with dolphin detector. Three-dimensional dose reconstruction of the treatment planning system calculation (TC) (A), COMPASS independent calculation (CC) (B), and COMPASS reconstruction from the dolphin detector measurement (CR) (C). Gamma results by comparing 3D reconstructed dose distribution of TC to CC (D), and TC to CR (F). Dose-volume histograms (DVHs) of TC and CC (E), and TC and CR (G). The orange, pink, brown, and blue lines are planning target volume, clinical target volume, rectum, and bladder, respectively. The dashed line represents the DVHs from TC, and solid line represents the DVHs from CC (E) or CR (G).

compared with those with criteria of $3 \% / 3 \mathrm{~mm}$ using paired t-tests. DVH deviations regarding clinically relevant errors were evaluated: the $\mathrm{DD}_{98 \%}(\%), \mathrm{DD}_{2 \%}(\%)$, and $\mathrm{DD}_{\text {mean }}(\%)$ for target volumes; the $\mathrm{DD}_{2 \%}(\%)$ and $\mathrm{DD}_{\text {mean }}(\%)$ for rectum and bladder. The $\mathrm{DD}_{98 \%}(\%)$ and $\mathrm{DD}_{2 \%}(\%)$ were defined as the percentage difference in the dose received by $98 \%$ and $2 \%$ volumes of structure. The $\mathrm{DD}_{\text {mean }}(\%)$ was defined as the percentage difference in the mean dose of the structure. Correlation between gamma results and DVH deviations was examined, and Pearson correlation coefficient $>0.8(p<0.05)$ was considered to be significant. All statistical tests were performed at 5\% level of significance using SPSS version 21 (IBM SPSS Statistics, Armonk, NY, USA).

\section{Results}

\section{Gamma results}

The structure-specific GFR and $\gamma$ Avg are shown in Tables 1 and 2.
The GFR in 2\%/2 mm criteria ranged up to 7.45\%. When TC and CC were compared, the mean GFR in 2\%/2 $\mathrm{mm}$ criteria for PTV, CTV, rectum, and bladder were $0.18 \%, 0.10 \%, 1.41 \%$, and $1.23 \%$, respectively. In comparison between TC and $\mathrm{CR}$, the values were $3.34 \%, 4.04 \%, 0.38 \%$, and $2.28 \%$, respectively. The GFR in $3 \% / 3$ $\mathrm{mm}$ criteria were less than $1 \%$ for all structures in all comparisons between TC, CC, and CR. The mean GFRs in 3\%/3 mm criteria were less than $0.1 \%$. In comparison between TC and CR, GFR in 2\%/2 $\mathrm{mm}$ criteria were significantly higher than those in $3 \% / 3 \mathrm{~mm}$ criteria for all structures.

The mean $\gamma$ Avg in 2\%/2 mm criteria for PTV, CTV, rectum, and bladder were $0.31,0.36,0.34$, and 0.40 , respectively, in comparison between TC and CC. In comparison between TC and CR, these values were $0.46,0.52,0.38$, and 0.40 , respectively. The $\gamma \mathrm{Avg}$ in the $2 \% / 2 \mathrm{~mm}$ criteria ranged up to 0.66 , and were significantly higher than those in the $3 \% / 3 \mathrm{~mm}$ criteria (which ranged up to 0.46 ) for all structures in all comparisons between $\mathrm{TC}_{1} \mathrm{CC}$, and $\mathrm{CR}$. 
Table 1. Three-dimensional dose reconstruction based, structure-specific gamma failure rates in 2\%/2 $\mathrm{mm}$ and $3 \% / 3 \mathrm{~mm}$ criteria

\begin{tabular}{|c|c|c|c|}
\hline & \multicolumn{2}{|c|}{ Gamma failure rate } & \multirow{2}{*}{$p$-value } \\
\hline & $2 \% / 2 \mathrm{~mm}$ & $3 \% / 3 \mathrm{~mm}$ & \\
\hline \multicolumn{4}{|l|}{ PTV } \\
\hline TC vs. CC & $0.18 \pm 0.55(0.00-2.81)$ & $0.00 \pm 0.00(0.00-0.01)$ & 0.093 \\
\hline TC vs. CR & $3.34 \pm 1.38(1.15-5.99)$ & $0.03 \pm 0.45(0.00-0.19)$ & $<0.001^{*}$ \\
\hline CC vs. CR & $2.34 \pm 1.52(0.00-5.74)$ & $0.08 \pm 0.16(0.00-0.75)$ & $<0.001^{*}$ \\
\hline \multicolumn{4}{|l|}{ CTV } \\
\hline TC vs. CC & $0.10 \pm 0.29(0.00-1.34)$ & $0.00 \pm 0.00(0.00-0.00)$ & 0.065 \\
\hline TC vs. CR & $4.04 \pm 2.27(0.19-7.45)$ & $0.01 \pm 0.03(0.00-0.16)$ & $<0.001^{\circ}$ \\
\hline CC vs. CR & $0.67 \pm 0.85(0.00-3.98)$ & $0.02 \pm 0.07(0.00-0.37)$ & $<0.001^{*}$ \\
\hline \multicolumn{4}{|l|}{ Rectum } \\
\hline TC vs. CC & $1.41 \pm 1.66(0.00-7.26)$ & $0.05 \pm 0.12(0.00-0.46)$ & $<0.001^{*}$ \\
\hline TC vs. CR & $0.38 \pm 0.70(0.00-3.25)$ & $0.02 \pm 0.05(0.00-0.19)$ & $0.007^{*}$ \\
\hline CC vs. CR & $2.51 \pm 1.64(0.15-6.61)$ & $0.01 \pm 0.03(0.00-0.14)$ & $<0.001^{*}$ \\
\hline \multicolumn{4}{|l|}{ Bladder } \\
\hline TC vs. CC & $1.23 \pm 1.14(0.00-4.72)$ & $0.07 \pm 0.10(0.00-0.41)$ & $<0.001^{*}$ \\
\hline TC vs. CR & $2.28 \pm 1.93(0.00-5.91)$ & $0.03 \pm 0.15(0.00-0.80)$ & $<0.001^{*}$ \\
\hline CC vs. CR & $1.02 \pm 1.06(0.00-3.99)$ & $0.00 \pm 0.00(0.00-0.00)$ & $<0.001^{*}$ \\
\hline
\end{tabular}

Values are presented as mean \pm standard deviation (range).

PTV, planning target volume; CTV, clinical target volume; TC, treatment planning system calculation; CC, COMPASS independent calculation; CR, COMPASS reconstruction from the dolphin detector measurement. $\mathrm{p}<0.05$.

Table 2. Three-dimensional dose reconstruction based, structure-specific average gamma value in $2 \% / 2 \mathrm{~mm}$ and $3 \% / 3 \mathrm{~mm} \mathrm{criteria}$

\begin{tabular}{|c|c|c|c|}
\hline & \multicolumn{2}{|c|}{ Average gamma value } & \multirow{2}{*}{$\mathrm{p}$-value } \\
\hline & $2 \% / 2 \mathrm{~mm}$ & $3 \% / 3 \mathrm{~mm}$ & \\
\hline \multicolumn{4}{|l|}{ PTV } \\
\hline TC vs. CC & $0.31 \pm 0.10(0.10-0.49)$ & $0.22 \pm 0.06(0.13-0.33)$ & $<0.001^{*}$ \\
\hline TC vs. CR & $0.46 \pm 0.41(0.37-0.51)$ & $0.31 \pm 0.04(0.24-0.39)$ & $<0.001^{*}$ \\
\hline CC vs. CR & $0.34 \pm 0.04(0.27-0.42)$ & $0.23 \pm 0.02(0.18-0.28)$ & $<0.001^{*}$ \\
\hline \multicolumn{4}{|l|}{ CTV } \\
\hline TC vs. CC & $0.36 \pm 0.12(0.17-0.57)$ & $0.23 \pm 0.09(0.00-0.38)$ & $<0.001^{*}$ \\
\hline TC vs. CR & $0.52 \pm 0.07(0.36-0.62)$ & $0.35 \pm 0.06(0.24-0.46)$ & $<0.001^{*}$ \\
\hline CC vs. CR & $0.29 \pm 0.09(0.00-0.46)$ & $0.20 \pm 0.04(0.14-0.30)$ & $<0.001^{*}$ \\
\hline \multicolumn{4}{|l|}{ Rectum } \\
\hline TC vs. CC & $0.34 \pm 0.05(0.21-0.46)$ & $0.23 \pm 0.03(0.16-0.30)$ & $<0.001^{*}$ \\
\hline TC vs. CR & $0.38 \pm 0.04(0.30-0.46)$ & $0.26 \pm 0.03(0.20-0.35)$ & $<0.001^{*}$ \\
\hline CC vs. CR & $0.48 \pm 0.06(0.35-0.58)$ & $0.32 \pm 0.04(0.23-0.39)$ & $<0.001^{*}$ \\
\hline \multicolumn{4}{|l|}{ Bladder } \\
\hline TC vs. CC & $0.40 \pm 0.08(0.12-0.59)$ & $0.28 \pm 0.05(0.20-0.39)$ & $<0.001^{*}$ \\
\hline TC vs. CR & $0.40 \pm 0.09(0.23-0.66)$ & $0.27 \pm 0.07(0.15-0.46)$ & $<0.001^{*}$ \\
\hline CC vs. CR & $0.34 \pm 0.07(0.20-0.44)$ & $0.23 \pm 0.05(0.14-0.29)$ & $<0.001^{*}$ \\
\hline
\end{tabular}

Values are presented as mean \pm standard deviation (range).

PTV, planning target volume; CTV, clinical target volume; TC, treatment planning system calculation; CC, COMPASS independent calculation; CR, COMPASS reconstruction from the dolphin detector measurement.

\section{DVH deviations}

DVH deviations in each structure are shown in Table 3. For PTV and $\mathrm{CTV}, \mathrm{DD}_{98 \%}, \mathrm{DD}_{2 \%}$ and $\mathrm{DD}_{\text {mean }}$ were less than $\pm 2 \%$ in all comparisons between $\mathrm{TC}, \mathrm{CC}$, and $\mathrm{CR}$. The mean $\mathrm{DD}_{98 \%}, \mathrm{DD}_{2 \%}$, and $\mathrm{DD}_{\text {mean }}$ for
PTV were $-0.42 \%, 0.61 \%$, and $0.57 \%$ in comparison between TC and $\mathrm{CC}$, and $-0.85 \%, 0.88 \%$, and $0.49 \%$ in comparison between $\mathrm{TC}$ and $\mathrm{CR}$, respectively.

For rectum and bladder, the $\mathrm{DD}_{2 \%}$ values were less than $\pm 2 \%$ in 
Table 3. Three-dimensional dose reconstruction based, structure-specific dose-volume histogram deviations

\begin{tabular}{lccc}
\hline & $\mathrm{DD}_{98 \%}(\%)$ & $\mathrm{DD}_{2 \%}(\%)$ & $\mathrm{DD}_{\text {mean }}(\%)$ \\
\hline PTV & $-0.42(-0.64,-0.20)$ & $0.61(0.43,0.79)$ & $0.57(0.46,0.68)$ \\
TC vs. CC & $-0.85(-0.98,-0.72)$ & $0.88(0.73,1.03)$ & $0.49(0.37,0.61)$ \\
TC vs. CR & $-0.42(-0.61,-0.23)$ & $0.26(0.10,0.42)$ & $-0.08(-0.22,0.06)$ \\
CC vs. CR & & & \\
CTV & $0.53(0.39,0.67)$ & $0.75(0.63,0.87)$ & $0.72(0.60,0.84)$ \\
TC vs. CC & $0.12(-0.02,0.26)$ & $1.18(1.07,1.29)$ & $0.92(0.82,1.02)$ \\
TC vs. CR & $-0.40(-0.55,-0.25)$ & $0.42(0.32 .0 .52)$ & $0.21(0.08,0.34)$ \\
CC vs. CR & - & $0.43(0.30,0.56)$ & $-1.09(-1.35,-0.83)$ \\
Rectum & - & $0.81(0.68,0.94)$ & $1.28(1.01,1.55)$ \\
TC vs. CC & - & $0.39(0.26,0.52)$ & $2.40(1.97 .2 .83)$ \\
TC vs. CR & - & & $-1.33(-1.95,0.71)$ \\
CC vs. CR & - & $0.75(0.61,0.89)$ & $-0.39(-1.09,0.31)$ \\
Bladder & - & $1.10(0.94,1.26)$ & $0.96(0.58,1.34)$ \\
TC vs. CC & - & $0.35(0.19,0.51)$ & \\
TC vs. CR & - & & \\
CC vs. CR & - & & \\
\hline
\end{tabular}

Values are presented as mean (95\% confidence interval).

PTV, planning target volume; CTV, clinical target volume; TC, treatment planning system calculation; CC, COMPASS independent calculation; CR, COMPASS reconstruction from the dolphin detector measurement; $\mathrm{DD}_{98 \%}(\%)$, percentage difference in dose received by $98 \%$ volume of structure; $\mathrm{DD}_{2 \%}(\%)$, percentage difference in dose received by $2 \%$ volume of structure; $\mathrm{DD}_{\text {mean }}(\%)$, percentage difference in mean dose of structure.

all comparisons between $\mathrm{TC}, \mathrm{CC}$, and $\mathrm{CR}$. The mean $\mathrm{DD}_{2 \%}$ for rectum and bladder were $0.43 \%$ and $0.75 \%$, respectively, in comparisons between TC and CC. In comparison between TC and CR, these values were $0.81 \%$ and $1.10 \%$, respectively. The mean $\mathrm{DD}_{\text {mean }}$ for rectum and bladder were $-1.09 \%$ and $-1.33 \%$, respectively, in comparison between TC and CC; for comparison between TC and $C R$, these values were $1.28 \%$ and $-0.39 \%$, respectively.

\section{Correlation analysis}

There was no strong correlation between the gamma results and DVH deviations for any structure, except for CTV (Table 4). For CTV, Pearson correlation coefficient was 0.961 ( $p<0.001$ ) between the $\gamma A v g$ in the $2 \% / 2 \mathrm{~mm}$ criteria and the $\left|D_{\text {mean }}\right|$, and 0.974 ( $p<$ 0.001 ) between the $\gamma \mathrm{Avg}$ in the $3 \% / 3 \mathrm{~mm}$ criteria and the $\left|D D_{\text {mean }}\right|$.

\section{Discussion and Conclusion}

In this study, 3D dose reconstruction-based pretreatment verification of VMAT plans for prostate cancer was performed. Both the independent calculation with the collapsed cone algorithm and the measurement with dolphin detector were used to validate the TPS calculation. The independent calculation has been used in many centers as another calculation that may reveal errors in TPS, and is generally less time consuming than measurement based verification [9]. However, independent calculations may include errors due to the known uncertainties of the model-based algo-
Table 4. Pearson correlation coefficient between structure-specific gamma results and DVH deviations ${ }^{a)}$

\begin{tabular}{|c|c|c|c|c|c|}
\hline Structures & $\begin{array}{l}\text { Acceptance } \\
\text { criteria }\end{array}$ & & $\left|\mathrm{DD}_{98 \%}(\%)\right|$ & $\mathrm{DD}_{2 \%}(\%)$ & $\mathrm{DD}_{\text {mean }}(\%)$ \\
\hline \multirow[t]{4}{*}{ PTV } & \multirow[t]{2}{*}{$2 \% / 2 \mathrm{~mm}$} & GFR & -0.245 & 0.293 & 0.381 \\
\hline & & $\gamma$ Avg & -0.551 & 0.516 & 0.630 \\
\hline & \multirow[t]{2}{*}{$3 \% / 3 \mathrm{~mm}$} & GFR & 0.361 & -0.509 & -0.153 \\
\hline & & $\gamma$ Avg & -0.707 & 0.641 & 0.687 \\
\hline \multirow[t]{4}{*}{ CTV } & \multirow[t]{2}{*}{$2 \% / 2 \mathrm{~mm}$} & GFR & 0.243 & 0.726 & 0.754 \\
\hline & & $\gamma$ Avg & 0.426 & 0.787 & $0.961^{*}$ \\
\hline & \multirow[t]{2}{*}{$3 \% / 3 \mathrm{~mm}$} & GFR & 0.010 & 0.048 & -0.038 \\
\hline & & $\gamma$ Avg & 0.505 & $0.836^{*}$ & $0.974^{*}$ \\
\hline \multirow[t]{4}{*}{ Rectum } & \multirow[t]{2}{*}{$2 \% / 2 \mathrm{~mm}$} & GFR & - & 0.255 & 0.220 \\
\hline & & $\gamma$ Avg & - & 0.474 & 0.552 \\
\hline & \multirow[t]{2}{*}{$3 \% / 3 \mathrm{~mm}$} & GFR & - & 0.168 & 0.011 \\
\hline & & $\gamma \operatorname{Avg}$ & - & 0.551 & 0.532 \\
\hline \multirow[t]{4}{*}{ Bladder } & \multirow[t]{2}{*}{$2 \% / 2 \mathrm{~mm}$} & GFR & - & 0.433 & 0.207 \\
\hline & & $\gamma$ Avg & - & 0.162 & 0.545 \\
\hline & \multirow[t]{2}{*}{$3 \% / 3 \mathrm{~mm}$} & GFR & - & 0.274 & -0.141 \\
\hline & & $\gamma$ Avg & - & 0.236 & 0.489 \\
\hline
\end{tabular}

DVH, dose-volume histogram; PTV, planning target volume; CTV, clinical target volume; $\mathrm{DD}_{98 \%}(\%)$, percentage difference in dose received by $98 \%$ volume of structure; $\mathrm{DD}_{20}(\%)$, percentage difference in dose received by $2 \%$ volume of structure; $\mathrm{DD}_{\text {mean }}(\%)$, percentage difference in mean dose of structure; GFR, gamma failure rate; $\gamma$ Avg, average gamma value.

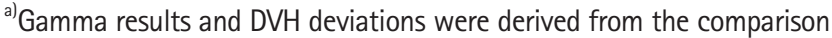
between treatment planning system calculation dose and COMPASS reconstruction from the dolphin detector measurement.

"Pearson correlation coefficient $>0.8$ and $p<0.05$. 
rithm [11]. In addition, errors in the actual delivery of VMAT plans are not validated by the independent calculation, and measurement based verification is also necessary.

We found that there was little discrepancy between the planned and independently calculated doses, as well as between the planned and measured doses. The TPS calculation by the Anisotropic Analytic Algorithm showed good agreement with the independent calculation using the collapsed cone algorithm in the COMPASS dose engine. The collapsed cone algorithm uses a 3D convolution superposition model, and is one of the most advanced calculation algorithms [12,13]. Chopra et al. [13] evaluated the accuracy of five dose calculation algorithms, and found that the collapsed cone algorithm along with the Acuros XB and Monte Carlo-based algorithms correlated best with measured data in both heterogeneous phantom and homogeneous phantom. In contrast, the Anisotropic Analytic Algorithm was reported to be less sensitive to tissue heterogeneities than the collapsed cone algorithm [12-14]. Differences between the Anisotropic Analytic Algorithm and the measured values were 3\%-5\% and 1.5\%-3.5\% in heterogeneous lung phantom and heterogeneous bone phantom, respectively, despite the excellent agreement between the Anisotropic Analytic Algorithm and the measured values in the homogeneous phantom [13]. However, our results found little discrepancy between the TPS calculation using the Anisotropic Analytic Algorithm and the independent calculation with the collapsed cone algorithm. This little discrepancy may be for several reasons; firstly, the calculated and measured regions of the VMAT plans consisted of relatively homogenous materials as the treatment volume included prostate and seminal vesicles or the surgical bed, excluding the regional lymphatic area. Therefore, in heterogeneous tissues which include air, such as the head and neck, or lung, the comparison between the Anisotropic Analytic Algorithm and collapsed cone algorithm may differ from the results found here. Because of this, further studies are needed in these types of cancer. Secondly, the degree of modulation in VMAT plans for prostate cancers can also be lower than that for other cancers due to the small treatment field size and less irregular shape of the target volume. Although there has been no concern about the cancer- or structure-specific acceptance criteria for gamma analysis, the degree of modulation may affect the quantity and magnitude of errors, and several studies have reported similar results to ours [14-16]. In the study by Vieillevigne et al. [16], which performed pretreatment verification for VMAT plans using three different detectors in 15 cases of prostate cancer, the GFRs were less than $2 \%$ and less than $7 \%$ for the acceptance criteria of $3 \% / 3 \mathrm{~mm}$ and $2 \% / 2 \mathrm{~mm}$ in all detectors, respectively. Thirdly, the accuracy of beam modeling in TPS might be improved by using a more recent version of TPS than that used in previous studies.

As the degree of clinically relevant errors may vary depending on the type of cancer, TPS and the delivery system used as well as the dosimetric measurement and analysis tools for pretreatment evaluation, cancer- and institution-specific criteria for pretreatment verification seems to be important. The 3D dose reconstruction-based pretreatment verification provides more thorough information on the quantity, magnitude, and clinical impacts of potential errors (such as under- or over-dosage than the TPS calculation). However, previous studies have mainly focused on the correlation analysis between gamma results and DVH deviations. Similar to our findings, previous studies reported weak or absence of correlation between GFR and DVH deviations in VMAT [17-20] and IMRT [21-24] plans for prostate cancers [17,18,20,23], as well as for other cancers [19-24]. Fundamentally, clinically relevant DVH parameters are extracted from the statistical analyses for the entire dose distribution, despite gamma results evaluating dose differences point by point. Weak or absence of correlation between GFR and DVH deviations is therefore likely inevitable regardless of cancer types, plans, and normalization methods, and additional evaluations for DVH parameters are needed to detect clinically relevant errors.

In the present study, we tried to evaluate gamma results with two acceptance criteria as well as DVH deviation in prostate cancer VMAT plans. Our results showed that the $2 \% / 2 \mathrm{~mm}$ criteria was more appropriate than the $3 \% / 3 \mathrm{~mm}$ criteria in VMAT plans for prostate cancer, as the $3 \% / 3 \mathrm{~mm}$ criteria might not be stringent enough to detect any discrepancies between the planned and measured dose distributions. There are several limitations to the present study. First, plans with intended errors were not evaluated. Second, pretreatment verification was performed with the COMPASS system with the dolphin detector only. There are several commercially available tools for 3D dose reconstruction based pretreatment verification, and gamma results and DVH deviations can be affected by spatial resolution of the detector, as well as by interpolation and the calculation algorithm [10,25]. Third, unlike the IMRT plans that use fixed gantry angles, VMAT plans cannot be evaluated by perpendicular field-by-field measurement, and the composite measurement used here may mask errors due to the summation of errors [10]. Fourth, dose errors in the low dosimetric regions can also be underestimated due to the global normalization, which evaluates dose differences based on the same value (the maximum planned dose) for all points. Fifth, 3D reconstructed dose distribution from the dolphin detector has an inherent limitation because COMPASS dose engine uses collapsed cone algorithm to calculate 3D dose from the measured 2D fluence map.

Although there have been no practical guidelines for the inter- 
pretation of DVH deviations, the target volume- or structure-specific DVH deviation may be very useful tools in the dosimetric evaluation for comparison between the planned and measured dose distribution. Our analysis showed that a dose difference in 98\%, 2\%, and mean volume of PTV, CTV, rectum, and bladder were within $\pm 2 \%$ between TC, CC, and CR. Further study for the proper dosimetric objects and appropriate tolerances in analysis of DVH deviations is needed.

In conclusions, the $3 \% / 3 \mathrm{~mm}$ criteria were not stringent enough to identify any discrepancies between the planned and measured dose, and DVH deviations were less than $2 \%$ in most parameters. Therefore, the gamma criteria of 2\%/2 $\mathrm{mm}$ and DVH related parameters could be useful tools in the pretreatment verification for VMAT in prostate cancer.

\section{Conflict of Interest}

No potential conflict of interest relevant to this article was reported.

\section{Acknowledgements}

This paper was supported by Wonkwang Institute of Clinical Medicine $\ln 2016$.

\section{References}

1. Bekelman JE, Rumble RB, Freedland SJ. Clinically localized prostate cancer: ASCO Clinical Practice Guideline Endorsement of an AUA/ASTRO/SUO Guideline Summary. J Oncol Pract 2018;14: 618-24.

2. Nguyen $\mathrm{PL}, \mathrm{Gu}$ X, Lipsitz $\mathrm{SR}$, et al. Cost implications of the rapid adoption of newer technologies for treating prostate cancer. J Clin Oncol 2011;29:1517-24.

3. Khan FM, Gibbons JP. Khan's the physics of radiation therapy. 5th ed. Philadelphia, PA: Lippincott Williams \& Wilkins; 2014.

4. Hatano K, Tohyama N, Kodama T, Okabe N, Sakai M, Konoeda K. Current status of intensity-modulated radiation therapy for prostate cancer: History, clinical results and future directions. Int J Urol 2019;26:775-84.

5. Cho B. Intensity-modulated radiation therapy: a review with a physics perspective. Radiat Oncol J 2018;36:1-10.

6. Low DA, Harms WB, Mutic S, Purdy JA. A technique for the quantitative evaluation of dose distributions. Med Phys 1998; 25:656-61.

7. Muzik J, Soukup M, Alber M. Comparison of fixed-beam IMRT, helical tomotherapy, and IMPT for selected cases. Med Phys
2008;35:1580-92.

8. Ezzell GA, Burmeister JW, Dogan N, et al. IMRT commissioning: multiple institution planning and dosimetry comparisons, a report from AAPM Task Group 119. Med Phys 2009;36:5359-73.

9. Low DA, Moran JM, Dempsey JF, Dong L, Oldham M. Dosimetry tools and techniques for IMRT. Med Phys 2011;38:1313-38.

10. Miften $M$, Olch A, Mihailidis D, et al. Tolerance limits and methodologies for IMRT measurement-based verification OA: recommendations of AAPM Task Group No. 218. Med Phys 2018;45: e53-e83.

11. Alber M, Mijnheer B, Georg D, et al. Guidelines for the verification of IMRT. Brussels, Belgium: European Society for Therapeutic Radiology and Oncology; 2008.

12. Vangvichith $M$, Autret $D$, Tiplica T, Barreau M, Dufreneix S. Comparison of five dose calculation algorithms in a heterogeneous media using design of experiment. Phys Med 2019;61:103-11.

13. Chopra $K L$, Leo $P$, Kabat $C$, et al. Evaluation of dose calculation accuracy of treatment planning systems in the presence of tissue heterogeneities. Ther Radiol Oncol 2018;2:420-7.

14. Kathirvel M, Subramanian S, Clivio A, et al. Critical appraisal of the accuracy of Acuros-XB and Anisotropic Analytical Algorithm compared to measurement and calculations with the compass system in the delivery of RapidArc clinical plans. Radiat Oncol 2013;8:140.

15. Kim JI, Choi CH, Wu HG, Kim JH, Kim K, Park JM. Correlation analysis between 2D and quasi-3D gamma evaluations for both intensity-modulated radiation therapy and volumetric modulated arc therapy. Oncotarget 2017;8:5449-59.

16. Vieillevigne L, Molinier J, Brun T, Ferrand R. Gamma index comparison of three VMAT QA systems and evaluation of their sensitivity to delivery errors. Phys Med 2015;31:720-5.

17. Sdrolia A, Brownsword KM, Marsden JE, Alty KT, Moore CS, Beavis AW. Retrospective review of locally set tolerances for VMAT prostate patient specific QA using the COMPASS system. Phys Med 2015;31:792-7.

18. Cozzolino M, Oliviero C, Califano G, et al. Clinically relevant quality assurance (QA) for prostate RapidArc plans: gamma maps and DVH-based evaluation. Phys Med 2014;30:462-72.

19. Jin X, Yan H, Han C, Zhou Y, Yi J, Xie C. Correlation between gamma index passing rate and clinical dosimetric difference for pre-treatment 2D and 3D volumetric modulated arc therapy dosimetric verification. Br J Radiol 2015; 88:20140577.

20. Park JM, Kim Jl, Park SY, Oh DH, Kim ST. Reliability of the gamma index analysis as a verification method of volumetric modulated arc therapy plans. Radiat Oncol 2018; 13:175.

21. Nelms BE, Zhen H, Tome WA. Per-beam, planar IMRT OA passing rates do not predict clinically relevant patient dose errors. Med 
Phys 2011; 38:1037-44.

22. Zhen $\mathrm{H}$, Nelms BE, Tome WA. Moving from gamma passing rates to patient DVH-based QA metrics in pretreatment dose QA. Med Phys 2011; 38:5477-89.

23. Stasi M, Bresciani $S$, Miranti A, Maggio A, Sapino V, Gabriele P. Pretreatment patient-specific IMRT quality assurance: a correlation study between gamma index and patient clinical dose volume histogram. Med Phys 2012; 39:7626-34.
24. Sun WZ, Zhang DD, Peng YL, et al. Retrospective dosimetry study of intensity-modulated radiation therapy for nasopharyngeal carcinoma: measurement-guided dose reconstruction and analysis. Radiat Oncol 2018; 13:42.

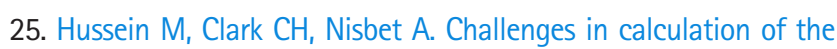
gamma index in radiotherapy: towards good practice. Phys Med 2017; 36:1-11. 